\title{
Antibiotic Usage and its Sensitivity Pattern in the NICU
}

\author{
Shrestha $\mathrm{R},{ }^{1}$ Shrestha JM, ${ }^{1}$ Gurung B
}

${ }^{1}$ Department of Pharmacology

${ }^{2}$ Department of Microbiology

Kathmandu University School of Medical Sciences (KUSMS)

Corresponding author

Ronak Shrestha

Department of Pharmacology

Kathmandu University School of Medical Sciences

Chaukot, Kavre

Email: ronakshrestha@gmail.com

Citation

Shrestha R, Shrestha JM, Gurung B. Antibiotic Usage and its Sensitivity Pattern in the NICU . Kathmandu Univ Med J 2012;38(2):27-32.

\begin{abstract}
Background

Sepsis is a major cause of mortality and morbidity in newborns affecting both developed and developing countries accounting a quarter around one million deaths per annum.
\end{abstract}

\section{Objective}

This study was aimed to assess the prevalence of neonatal sepsis, culture isolates, pattern, antibiotic sensitivity and drug prescribing pattern.

\section{Methods}

In present prospective study, 48 neonates admitted in pediatric ward from January to March 2011 of Dhulikhel Hospital were included. The gestation age, onset of sepsis, culture isolates, antibiotic sensitivity pattern and drug prescribing pattern were studied. Environmental air sampling of NICU (Neonatal Intensive Care Unit) was done by settle plate method for microbial examination.

\section{Result}

Among 48 neonates, 23 (47.92\%) neonates were culture positive with predominant isolates of Klebsiella oxytoca 11(47.83\%), Pseudomonas spp. 4(17.39\%), Methicillin Resistant Staphylococcus Aureus (MRSA) 3(13.04\%) and single case of Enterobacter spp. In environmental samples, K. oxytoca and Enterobacter spp. were isolated. Amikacin, Imepeneum and Ciprofloxacin were sensitive whereas Ampicillin in combination with Cloxacillin, Ampicillin, Aztreonam were resistant for Klebsiella. oxytoca. Amikacin, Ceftazidime and Imipenuem are sensitive to Enterobacter spp. The single or combinations of resistant antibiotics were found to be prescribed.

\section{Conclusion}

The emerging antibiotic resistances among the culture isolates and coherence with environmental samples were observed. Hence, special measures are imperative for reducing environmental contamination and the rational usage of antibiotics for preventing the infection and emerging antibiotic resistance. The study recommends need of the antibiotic policy to curb the present scenario.

\section{KEY WORDS}

Antibiotic Sensitivity, Neonatal sepsis, NICU

\section{INTRODUCTION}

Health care associated infections are an important problem in neonatal intensice care unit (NICU) in which environmental and host factors often contribute to higher rates of infections. Infections with Gram Negative Bacilli that are resistant to many commonly used antibacterial drugs are increasing reported in NICUs. ${ }^{1}$ Manifestations of neonatal sepsis are non-specific. A high indicator of suspicion with or without laboratory evidences of infection is the key for early diagnosis.

Neonatal sepsis refers to the infection occurring within the neonatal period i.e. the first 28 days of life, and up to four weeks beyond the expected date of delivery in a preterm baby. Sepsis is broadly classified into early- or late-onset sepsis. Early onset sepsis is present at birth or presents within the first 48 hours of life. The most common infecting organisms are Group B Streptococcus (GBS) and E. coli and are mainly caused by vaginal or perineal organisms. The burden of gram negative pathogens such as Klebsiella, Pseudomonas and Acienobacter spp. accounts almost half of early onset neonatal infections in developing countries. ${ }^{2}$ Late onset sepsis is present after 48-72 hours of life and can 
be hospital or community acquired and sometimes may be also due to perinatally acquired organisms. The most prevalent causative organism for health care associated infections in NICU is Coagulase Negative Staphylococcus aureus (CONS) both in developed and developing countries. ${ }^{3}$ Hence, prompt management with antibiotic therapy and supportive care will serve as a benefit for cases of neonatal sepsis. Antibiotics are the commonest drugs used in the NICU and have undoubtedly played a role in their improved survival. The neonatologist needs the skill and knowledge to outweigh the risk and benefits of antibiotics. Clark et al reported that $98 \%$ of preterm infants who received empiric antibiotics were culture negative. ${ }^{4}$ However, the large use of antimicrobials drugs and cross transmission via the hands of care givers, contaminated equipments, or environment plays a prominent role in causation and dissemination of infections.

Hence, the purpose of the study is to access the prevalence of neonatal sepsis, culture isolates from the neonate's bloodstream and environment, and appropriate use of antibiotics in sepsis and its sensitivity test pattern.

\section{METHODS}

This was a prospective study carried out by department of pharmacology in pediatric ward of Dhulikhel Hospital of Nepal from January - March 2011. A total of 48 neonates who remained admitted in pediatric ward for at least 48 hours during the study period were enrolled. The gestation age, onset of sepsis, microbiological data about culture isolates from bloodstream and environmental air sampling were collected. Also, antibiotic sensitivity test data and antibiotic prescribed were collected.

\section{Microbiological surveillance:}

Bloodstream culture was done by collecting the blood sample $(3 \mathrm{ml})$ by aseptic measures and was dispensed in a Brain Heart Infusion (BHI) broth at a ratio of 1:10 for neonates. Then, it was incubated for $24-48$ hours at $37^{\circ} \mathrm{C}$ in an incubator. Further, turbitidity examination was conducted and inoculation was done in Blood agar plate, MacConkey agar incubation for 24 - 48 hours at $37^{\circ} \mathrm{C}$ in an incubator. Identification of bacteria by Gram staining, biochemical reactions and followed by antibiotic sensitivity test by disc diffusion method.

Likewise, environmental sampling was conducted according to settle plate method. The exposed plated were incubated for 24 hours at $37^{\circ} \mathrm{C}$. The colonies were counted and results were expressed as the number of bacteria-carrying particles (bcp) settling on a given area at a given period of time. ${ }^{5}$ For the purpose of our study, antibiotic sensitivity test was conducted by Kirby-Baure disc diffusion method. Results were compared with the standard charts provided by $\mathrm{Hi}-$ Media, India.

\section{Statistical analysis:}

The collected data were entered into Statistical Programme for Social science (SPSS) version 16 and analysis was done. Results were expressed as numbers and percentages for gestation age, onset of sepsis, culture isolates, and antibiotics prescribed. Environmental bioload were expressed in bacteria carrying paticles (bcp) per meter ${ }^{3}$. Chi-square test was conducted to show the association between gestation age and onset of sepsis. A p value less than 0.05 were considered statistically significant.

\section{RESULTS}

Among 48 cases that were included in the study, 37 (77.1\%) were term and 11 (22.9\%) were preterm babies. A total of $43(89.6 \%)$ of the cases had early onset sepsis and 5 (10.4\%) had late onset sepsis. Out of these, 18 (41.7\%) neonates were found to be culture positive. A total of 23 (47.9\%) organisms were isolated from the bloodstream of the neonates, among which Klebsiella oxytoca $11(47.8 \%)$ was the most common. A single case of Enterobacter spp., Aceinobacter spp. and Streptococcus spp. were also isolated (table 1). Organisms like Klebsiella oxytoca, Staphylococcus aureus (64 bcp/m3), and E.coli (40 bcp/m3) were isolated from the air samples of NICU and Pediatrics intensive care unit (PICU). Likewise, Enterobacter spp, E. coli, Pseudomonas spp, Methicillin Resistant Coagulase Negative Staphylococci (MRCONs) were isolated from equipments like suction tube, tap ambulatory bag and trolley that was found to be used in NICU with bioload 590 $\mathrm{bcp} / \mathrm{m3}$. Also, in PICU and Labor room MSSA, MRCONs and Pseudomonas spp. were isolated from trolley, nursing station table and water from oxygen humidifier with 80

Table 1. Different variables and types of bacterial isolates based on sepsis onset.

\begin{tabular}{|llll|}
\hline \multicolumn{2}{|c}{ Variables } & Total N (\%) & p-value \\
\hline Gestation age & Term & $37(77.1)$ & \\
& Preterm & $11(22.9)$ & \\
\hline \multirow{2}{*}{ Onset of sepsis } & Early-onset & $43(89.6)$ & 0.697 \\
& Late-onset & $5(10.4)$ & \\
\hline Culture positive & & $18(41.7)$ &
\end{tabular}

\begin{tabular}{|c|c|c|c|}
\hline \multirow{2}{*}{ Microorganism } & \multicolumn{2}{|c|}{ Onset of sepsis } & \multirow{2}{*}{$\begin{array}{l}\text { Total } \\
\text { N }\end{array}$} \\
\hline & Early-onset n (\%) & $\begin{array}{l}\text { Late-onset } \\
\text { n (\%) }\end{array}$ & \\
\hline Klebsiella oxytoca & $7(63.6)$ & $4(36.4)$ & 11 \\
\hline Pseudomonas & $2(50.0)$ & $2(50.0)$ & 4 \\
\hline MRSA & $3(100)$ & - & 3 \\
\hline MSSA & $2(100)$ & - & 2 \\
\hline Enterobacter spp. & - & $1(100)$ & 1 \\
\hline Aceinobacter spp. & $1(100)$ & - & 1 \\
\hline Streptrococcus spp & $1(100)$ & - & 1 \\
\hline
\end{tabular}


Table 2. Type of bacterial isolates from environment and equipment sampling.

\begin{tabular}{|c|c|c|c|c|c|}
\hline Sites & Microoragnisms & $\begin{array}{l}\text { Bioload } \\
\left(\mathrm{bcp} / \mathrm{m}^{3}\right)\end{array}$ & Sites & Microorganisms & $\begin{array}{l}\text { Bioload } \\
\left(\mathrm{bcp} / \mathrm{m}^{3}\right)\end{array}$ \\
\hline NICU \& PICU & & & Labor room & & \\
\hline \multirow[t]{3}{*}{ Entrance door } & ASB & 242 & Water oxygen humidifier & Pseudomonas spp & \\
\hline & Diptheroids & & Ambulatory bag & ASB & \\
\hline & S. aureus & & & & \\
\hline Procedure bed & S. aureus & & NICU & & 590 \\
\hline \multirow[t]{4}{*}{ Bed 1} & Micrococcus & 117 & Nursing station & No growth & \\
\hline & Diptheroids & & Ventilator & No growth & \\
\hline & ASB & & Bed linen & ASB & \\
\hline & S. citrus & & Water oxygen humidifier & ASB & \\
\hline \multirow[t]{3}{*}{ Bed 2} & Micrococcus & 71 & Oxygen mask & No growth & \\
\hline & S. citrus & & Suction tube & Enterobacter spp, E.coli & \\
\hline & ASB & & Tap & Pseudomonas spp & \\
\hline \multirow[t]{4}{*}{ Bed 3} & Klebsiella oxytoca & 64 & Ambulatory bag (inside) & MRCONs & \\
\hline & S. aureus & & Ambulatory bag (clean) & ASB & \\
\hline & S. citrus & & Trolley & MSCONs & \\
\hline & Micrococcus & & Spirit & No growth & \\
\hline \multirow[t]{3}{*}{ Bed 4} & ASB & 17 & Betadine & No growth & \\
\hline & Micrococcus & & Antibiotic pumper & No growth & \\
\hline & Diptheroids & & Heating filter & No growth & \\
\hline \multirow[t]{4}{*}{ Bed 5} & Fungus & 30 & Soap & ASB & \\
\hline & Diptheroids & & Ambulatory bag outside 1 & ASB & \\
\hline & Micrococcus & & Ambulatory bag outside 2 & ASB & \\
\hline & S. citrus & & & & \\
\hline \multirow[t]{3}{*}{ Bed 6} & ASB & 33 & PICU & & \\
\hline & Micrococcus & & Nursing station table & ASB, Diptheroids, S.aureus & 80 \\
\hline & Diptheroids & & Bed floor & ASB, Diptheroids, S.aureus & 80 \\
\hline \multirow[t]{3}{*}{ Bed 7} & E.coli & 40 & Trolley & MSSA & \\
\hline & Diptheroids & & Nursing station table & MRCONs & \\
\hline & S. citrus & & Water oxygen humdifier & ASB & \\
\hline \multirow[t]{4}{*}{ Bed 8} & Fungus & 91 & Oxygen mask & No growth & \\
\hline & Micrococcus & & Soap & No growth & \\
\hline & S. aureus & & Spirit & No growth & \\
\hline & S. citrus & & Ambulatory bag & ASB & \\
\hline
\end{tabular}

$\mathrm{bcp} / \mathrm{m} 3$ bioload respectively as shown in (table 2).

According to antibiotic sensitivity test, most of the antibiotics were resistant. For Klebsiella oxytoca, antibiotics like Amikacin, Ciprofloxacin and Imipenum were found to be sensitive. Imipenum was sensitive and Cefotaxime was found to be partially sensitive for Pseudomonas spp. Likewise, Amikacin, Gentamicin and Vancomycin was sensitive to MRSA. In the other hand, for Enterobacter and Aceinobacterspp. Ceftriaxone was found to be sensitive and antibiotics like Ciprofloxacin, Ceftriaxone and Vancomycin were found to be sensitive for Streptococcus spp. (table 3). Ampicillin in combination with Cloxacillin was found to be mostly prescribed antibacterial agent for all isolated organism such as Klebisalla oxytoca (72.7\%), Pseudomonas spp. (75.0\%), MRSA (66.7\%) and for all the single positive cases of MSSA, Enterobacter spp., and Aceinobacter spp. (100\%). Likewise, Amikacin was found to be highly prescribed for Klebisalla oxytoca (81.8\%), Pseudomonas spp. (50.0\%), MRSA (33.3\%), MSSA (100\%), Enterobacter spp. (100\%) and Streptococcus spp. (100\%). Also, Ceftazidime (36.4\%), Gentamicin and Imipenum (27.3\%) for Klebsiella oxytoca and Gentamicin and Imipenum (25.0\%) for Pseudomonas spp. Ciprofloxacin (50.0\%) was found to prescribed for single case (out of 2 cases) MSSA along with other antibiotics and Metronidazole (9.1\%) was also found to be prescribed for single case (out of 11 cases) of Klebsiella oxytoca as shown in (table 4).

\section{DISCUSSION}

In the present study, the prevalence of neonatal sepsis with positive blood culture was $41.7 \%$ with no significant 
Table 3. Antibitoic sensitivity pattern of commonly used antibiotics in the study.

\begin{tabular}{|c|c|c|}
\hline Oragnism & Sensitive antibiotic & Resistant antibiotic \\
\hline \multirow[t]{4}{*}{ Klebsiella } & Amikacin & Ampicillin + Cloxacillin \\
\hline & Ciprofloxacin & Ampicillin \\
\hline & Imipenum & Aztreonam \\
\hline & & Cefotaxime \\
\hline \multirow[t]{6}{*}{ Pseudomonas } & Imipenum & Ampicillin + Cloxacillin \\
\hline & Cefotaxime (PS) & Ampicillin \\
\hline & & Aztreonam \\
\hline & & Amikacin \\
\hline & & Gentamicin \\
\hline & & Ciprofloxacin \\
\hline \multirow[t]{5}{*}{ MRSA } & Amikacin & Ampicillin + Cloxacillin \\
\hline & Gentamicin & Ampicillin \\
\hline & Vancomycin & Aztreonam \\
\hline & & Cefotaxime \\
\hline & & Cloxacillin \\
\hline \multirow[t]{4}{*}{ MSSA } & Ampicillin & Ampicillin+Cloxacillin \\
\hline & Amikacin & Aztreonam \\
\hline & & Cefotaxime \\
\hline & & Gentamicin \\
\hline \multirow[t]{4}{*}{ Enterobacter } & Amikacin & Ampicillin+Cloxacillin \\
\hline & Imipenum & Aztreonam \\
\hline & Ceftazidime & Cefotaxime \\
\hline & & Gentamicin \\
\hline \multirow[t]{4}{*}{ Streptococcus } & Ampicillin & Amikacin \\
\hline & Cefotaxime & \\
\hline & Gentamicin & \\
\hline & Vancomycin & \\
\hline \multirow[t]{3}{*}{ Aceinobacter } & Ceftriaxone & Amikacin \\
\hline & Imipenum & Gentamicin \\
\hline & & Ciprofloxacin \\
\hline
\end{tabular}

difference in onset of sepsis. The study corresponds to the study conducted by Kayange $\mathrm{N}$ in Tanzania, where 39\% of the admitted cases have septicemia with no significant difference in onset of sepsis. ${ }^{6}$ In the current study, $89.6 \%$ of the cases had early-onset sepsis which is in coherence with the study done by Jain NK, et al in 2003 where $80 \%$ had early onset sepsis. ${ }^{7}$ The result also corresponds to the study conducted by Vinod Kumar et al. (2008) where the reported prevalence of early onset neonatal sepsis was high (73\%). ${ }^{8}$ Gram negative bacteria were more common than Gram positive bacteria in our study and these results were in concordance with some of the other studies Sundaram et al and Aletaye SMH et al. ${ }^{9,10}$ Our study is in consistent with the studies conducted by Boo NY and Zaidi AK where almost half of early-onset bloodstream infections in developing regions are due to Gram negative bacteria such as Klebsiella spp., Pseudomonas spp. and Acienobacter spp. ${ }^{11,12}$ In our study Klebsiella oxytoca which accounts $63.6 \%$ and $36.4 \%$ for early-onset and late-onset sepsis, was the most common microorganism isolated from the blood cultures. Klebsiella spp. is recognized as one of the most common neonatal pathogen in developing countries as shown by the study Jain NK in Nepal where Klebsiella spp. and E.coli were the most common organisms showing a coherence with our study. ${ }^{7}$ According to the study done by Joshi SG et al, among other Gram negative organisms; Pseudomonas spp, Aceinobacter spp. and Enterobacter spp. are likely to cause neonatal sepsis which had been also isolated in our study. ${ }^{13}$ In the study organisms like Klebsiella oxytoca, E.coli, S. aureus, Pseudomonas spp., Enterobacter spp., MRCONs, MSCONs and MSSA were isolated from environmental air sampling of NICU, PICU and labor room. Hence, the likelihood of resultant septicemia could be due to isolation of the same microorganisms from environmental sampling.

Antimicrobial resistance is a widespread global problem that has caused ineffectiveness of the current empirical treatment against microorganisms. Knowledge about

Table4. Prescribed antibiotics for isolated microorganisms.

\begin{tabular}{|c|c|c|c|c|c|c|c|}
\hline \multirow[b]{2}{*}{$\begin{array}{l}\text { Antibiotic pre- } \\
\text { scribed }\end{array}$} & \multicolumn{7}{|c|}{ Organism } \\
\hline & $\begin{array}{l}\text { Klebsiella } \\
\mathrm{N}(\%)\end{array}$ & $\begin{array}{l}\text { Pseudomonas } \\
\mathrm{N}(\%)\end{array}$ & $\begin{array}{l}\text { MRSA } \\
\text { N (\%) }\end{array}$ & $\begin{array}{l}\text { MSSA } \\
\text { N (\%) }\end{array}$ & $\begin{array}{l}\text { Enterobacter } \\
N(\%)\end{array}$ & $\begin{array}{l}\text { Aceinobacter } \\
\mathrm{N}(\%)\end{array}$ & $\begin{array}{l}\text { Streptococcus } \\
\mathrm{N}(\%)\end{array}$ \\
\hline Ampicillin+Cloxacillin & $8(72.7)$ & $3(75.0)$ & $2(66.7)$ & $2(100)$ & $1(100)$ & $1(100)$ & $1(100)$ \\
\hline Ampicillin & $4(36.4)$ & $1(25.0)$ & - & - & $1(100)$ & $1(100)$ & - \\
\hline Cloxacillin & - & - & - & - & - & - & - \\
\hline Amikacin & $9(81.8)$ & $2(50.0)$ & $1(33.3)$ & $2(100)$ & $1(100)$ & - & $1(100)$ \\
\hline Gentamicin & $3(27.3)$ & $1(25.0)$ & $1(33.3)$ & - & - & - & - \\
\hline Imipenum & $3(27.3)$ & $1(25.0)$ & - & $1(50)$ & - & - & - \\
\hline Cefotaxime & - & - & - & $1(50)$ & - & - & - \\
\hline Ceftazidime & $4(36.4)$ & - & - & - & - & - & - \\
\hline Ciprofloxacin & - & - & - & $1(50)$ & - & - & - \\
\hline Metronidazole & $1(9.1)$ & - & - & & - & - & - \\
\hline
\end{tabular}

Sample size (n): 11 Klebsiella oxytoca, 4 Pseudomonas spp., 3 MRSA, 2 MSSA, single species of Enterobacter, Aceinobacter and Streptococcus 
antimicrobial resistance and trends in resistance patterns among major microorganisms causing infections in young infants is an important component for management strategies. In our study, most of the Gram positive and Gram negative bacteria were resistant to antibiotics that were found to be commonly prescribed as (Ampicillin + Cloxacillin and Ampicillin), monobactums (Aztreonam) and aminoglycosides (Gentamicin) which is in agreement to similar studies by Lund et al, where most of the Gram positive and negative organisms were resistant to commonly prescribed antibiotics. ${ }^{14}$ This result is also in coherence with the study done by Zaidi AK et al, where the WHO recommended Ampicillin and Gentamicin for the treatment of neonatal sepsis may no longer be effective as $71 \%$ of Klebsiella spp. and $50 \%$ of E.coli are reportedly resistant to Gentamicin. ${ }^{15}$ However, these microorganisms were sensitive to Carbapenams (Imipenam) and fluoroquinolones (Ciprofloxacin). In the study, Gram negative organisms were resistant to penicillins as well resistant to third generation cephalosprins probably attributable to the extended spectrum $\beta$ - lactamase (ESBL) production, suggesting a clonal outbreak in hospital setting. As stated earlier, almost half of early-onset sepsis is due to Klebsiella, Pseudomonas and Aceinobater spp., which are the common sources of outbreak as they thrive in multi-use containers of medications, liquid soaps and other solutions, including antiseptics and disinfectants, and on inadequately reprocessed equipments. In our study, similar microorganisms were also isolated during environmental air sampling. Likewise, $S$. aureus remains a very important neonatal pathogen in developing countries with numerous reported outbreaks, was also isolated from the nursing station and bed floors of NICU and PICU which are the main source of spread according to Harris J. et al., 2001 suggesting the prospect of an outbreak of infectious microorganisms. ${ }^{16,17}$ The study is similar to the study done by Pessoa-Silva CL, Meurer Sampiao JL et al, where the incidence of infections caused by organisms resistant to $\beta$ - lactam agents has sharply increased in recent years and has often been associated to clonal outbreak which is also seen in our study..$^{18}$

As we observed in the current study, most of the Gram negative organisms were highly prescribed Amipicillin in combination with Cloxacillin (Klebsiella spp. 72.7\%, Pseudomonas spp. 75.0\%, and Aceinobacter spp. 100.0\%) and Gentamicin (Klebsiella spp. 27.3\%, Pseudomonas spp. $50.0 \%$ ) as an empiric treatment recommended by WHO, were resistant. However, sensitive antibiotics such as carbepenums (Imipenum), third generation cephalosporins (Cefotaxime) and fluoroquinolones (Ciprofloxacin) were prescribed which suggests a partial rational approach for the management of sepsis as shown in table 4. Likewise, for gram positive organisms' resistant antibiotics such as Ampicillin + Coxacillin (MRSA 66.7\%, every single positive case of MSSA and Streptococcus spp.) along with sensitive antibiotics aminoglycosides (Amikacin and Gentamicin) were prescribed showing a limited rationality for management of sepsis. Even though, management of neonatal sepsis was done as per WHO recommendation for the early management; it seems to be curtailed as most of the antibiotics prescribed were resistant showing imperative need of rational usage of antibiotics for preventing the infection and emerging antibiotic resistance.

\section{CONCLUSION}

Gram negative bacteria were the main cause of early and late-onset of sepsis. Klebsiella oxytoca was the most common pathogen isolated from the bloodstream of neonates and environment. Most of the isolated organisms were resistant to the prescribed antibiotics. Hence, the emerging antibiotic resistances among the culture isolates and coherence with environmental samples observed requires the need of special measures imperative for reducing environmental contamination and the rational usage of antibiotics for preventing the infection and emerging antibiotic resistance. At present, the study recommends need a greater understanding of the epidemiology of health care associated organisms and peculiar features of health care units and antibiotic policy to curb the present scenario.

\section{REFERENCES}

1. Caterina RP, Paola DC, Domenico C. Survillence of multidrug-resistant gram negative bacilli in a neonatal intensive care unit: prominent role of cross transmission. AJIC 2007;35 (4):222-230.

2. Zaida KMA, Huskins WC, Thaver D. Hospital -acquired neonatal infections in developing countries. Lancet 2005;365:1175-88.

3. Jacqueline J HO. Appropriate use of antibiotics in the NICU. Eastern Journal of Medicine 2010; 15:133-138.

4. Clark RH, Bloom BT, Spitzer AR, Gerstmann DR. Empiric use of ampicillin and cefotaxime, compared with ampicillin and gentamicin, for neonates at risk for sepsis in associated with an increased risk of neonatal death. Paedriatics 2006;117:67-74.

5. Mackie and McCartney. Practical Medical Microbiology. In: Gerald Colle et al. editors. Examination of water, food and air: Examination of air, settle plate method. 14th ed. New York, Churchill Livingstone. 1996; 906-8.

6. Kayange N, Kamugisha E, Mwizamholya L D. Predictors of positive blood culture and deaths among neonates with suspected neonatal sepsis in a tertiary hospital, Mwanza-Tanzania. BMC Paedriatics 2010; 10:39.

7. Jain NK, Jain VM, Maheshwari S. Clinical profile of Neonatal Sepsis. Kathmandu University Medical Journal 2003;1(2):117-120.

8. VinodKumar CS, Neelagubd YF, Seneeta K. Pernatal risk factors and microbiological profile of neonatal septicemia: A multicentred study. J. Obstet. Gynaecol. India 2008;58 (1):32-40.

9. Sundaram V, Kumar P, Dutta S, Mukhopadhyay K, Ray P, Vikas et.al. Blood Culture Confirmed Bacterial Sepsis in Neonates in a North Indian Tertiary Care Center: Changes over the Last Decade Jpn. J. Infect. Dis 2009;62(1): 46-50.

10. Aletayeb SMH, Dokht Khosravi AD, Dehdashtian M, Kompani $F$, Mortazavi SM and Aramesh MR. Identification of bacterial agents and antimicrobial susceptibility of neonatal sepsis: A 54-month study in a tertiary hospital. African Journal of Microbiology Research 2011; 5(5): 528-53. 
11. Boo NY, Chor CY. Six year trend of neonatal septicaemia in a large Malaysian maternity hospital. J Paediatr Child Health 1994; 30:23-27.

12. Zaidi AK, Ali SA, Darmstadt GL, Bhutta ZA. Explore simplified antimicrobial regimens for the treatment of neonatal sepsis. Meeting Report; 30th September-1st October 2002. Geneva: Department of Child and Adolescent Health and Development, World Health Organization, 2002.

13. Joshi SG, Ghole VS, Niphadhar KB. Neonatal gram negative bacteremia. Indian J Paedr 2000;67(1):27-32.

14. Lund AM, Christensen E, Skovby F (2002). Diagnosis and acute treatment of inborn metabolic diseases in infants. Ugeskrift for Laeger. 164(48): 5613-5619.

15. Zaida AK, Huskins WC, Thaver D. Hospital acquired neonatal infections in developing countries. Lancet 2005;365:1175-1188.
16. Bisson G, Fishman NO, Patel JB. Extended spectrum $\beta$-lactamse producing Escherichia coli and Klebsiella spp: risk factors for colonization and impact of antimicriobial formulary interventions on colonization prevalence. Infect control Hosp Epidemiol 2002;23:254260.

17. Harris J, Goldmann D. Infections acquired in the nursery: Epidemiology and control. In: Remington JS, Klein JO, eds. Infectious diseases of the fetus, newborn and infants. 5th ed. Philadelphia: WB Saunders, 2001: 1371-418.

18. Pessoa-Silva CL, Meurer Sampiao JI. Extended spectrum $\beta$-lactamase producing Klebsiella pneumoniae in a neonatal intensive care unit: risk factors for infection and colonization. J Hosp Infect 2003;53:198206. 